\title{
Internet use among patients with psychosomatic disorders: What are the health-related demands and needs?
}

Journal of Health Psychology 16(7) II 20-1 I 26

(C) The Author(s) 2011

Reprints and permissions:

sagepub.co.uk/journalsPermission.nav DOI: $10.1177 / 13591053$ |140167| hpq.sagepub.com

\author{
Martin Teufel', Norbert Schäffeler', \\ Martina de Zwaan ${ }^{2}$, Holmer Graap ${ }^{2}$, \\ Stephan Zipfel', Katrin Elisabeth Giel'
}

\begin{abstract}
While Internet-based health-related offers for various disorders are increasing, little is known about the way patients with psychosomatic disorders use the Internet. We conducted a questionnaire survey in outpatient clinics at two university hospitals' Departments of Psychosomatic Medicine. Seventy-four percent of N= 274 patients had Internet home access and 60 percent of those patients reported health-related Internet use. Patients expressed the wish for more professional, evidence-based online information and expert-guided treatment. The survey results support the Internet's high potential in future treatment strategies for patients with psychosomatic disorders.
\end{abstract}

\section{Keywords}

access, Internet, patients, psychosomatic, use

\section{Introduction}

The Internet is growing increasingly important with regard to health-related topics (Evers et al., 2003). In the context of online medical information and treatment, many recent studies have focused on access to and the quality of healthrelated Internet sources, the impact of Internet use on the patient-physician relationship and telemedical web-based treatment strategies.

In the last decade, a dramatic increase in Internet searches for health information has been shown (Trotter and Morgan, 2008). According to a survey by Diaz et al. (2002), about half of patients enrolled in a primary care practice use the Internet to access general medical information. Internet use increases for specific disease-related information, in particular, for severe and chronic somatic diseases (e.g. cancer).

\footnotetext{
'Medical University Hospital Tuebingen, Department of Psychosomatic Medicine and Psychotherapy, Germany. ${ }^{2}$ University Hospital Erlangen, Department of Psychosomatic Medicine and Psychotherapy, Germany.

\section{Corresponding author:}

Katrin Elisabeth Giel, PhD, Medical University Hospital Tuebingen, Department of Psychosomatic Medicine and Psychotherapy, Osianderstr. 5, 72076 Tuebingen, Germany. Email: katrin.giel@med.uni-tuebingen.de
} 
Despite patients' growing reliance on Internet health sites, scientists have rarely examined patients' access to the Internet or their demands on those web sources. While there are survey data on the Internet use of patients with somatic diseases, to our knowledge, no structured survey among patients with psychosomatic disorders has been conducted to date. Psychosomatic disorders include conditions that are characterized by a pronounced interaction of mental and somatic factors in the genesis or maintenance of the disorder, such as anxiety and mood disorders, eating and weight disorders, pain and somatoform disorders as well as psychological reactions to severe somatic diseases (adjustment disorders).

Recently, an increasing number of workgroups designed and evaluated therapeutic e-health programs. In psychosomatic medicine and psychotherapy, new web-based approaches to diagnosis and treatment include online surveys about patient and disease characteristics (Enck et al., 2006; Roberto et al., 2010), online delivered information and education (Simpson et al., 2009; Wisner et al., 2008), screening (Donker et al., 2009) and online psychotherapy (Kessler et al., 2009; Kiropoulos et al., 2008; Mitchell et al., 2008). Other online surveys address web-based self-help (Van't Hof et al., 2009) and aftercare programs (Golkaramnay et al., 2007), delivered either by a self-guided program or email, chat and recently, by video conferencing.

These telemedical treatment strategies are of particular economic and political interest. For example, telemedical treatment minimizes travel time. Travel burden is a key deterrent in accessing health care (Probst et al., 2007), especially in rare diseases and outpatient care. Patients in rural regions or in need of frequent physician contact can profit from these new technological developments (Marziali, 2009; Olsson et al., 2004; Van Straten et al., 2008). This is also true for patients with psychosomatic disorders. In most health-care systems, only a limited number of treatment centers specialize in treating these disorders. Hence, they are treating patients from a large area with travel times up to several hours, which makes telemedicine a highly relevant topic within psychosomatic medicine. A challenge to telemedicine as a psychotherapy treatment strategy is that new web-based programs have to face an altered therapeutic alliance and relationship. While it is a challenge for therapists and researchers in the field of psychosomatic medicine to develop feasible and effective treatment strategies, empathy through distant cyberspace seems to be possible (Teufel et al., 2009).

To successfully establish telemedical approaches and programs, it is necessary to acquire knowledge about Internet accessibility, characteristics of Internet use among patients, their wishes concerning new technologies, and patient Internet use in therapeutic contexts. However, such data are currently not available in the field of psychosomatic medicine. Our present survey closes the research gap by examining these questions using a sample of German patients with psychosomatic disorders.

\section{Materials and methods}

We carried out a survey in the outpatient units of the Departments of Psychosomatic Medicine and Psychotherapy at the Medical University Hospital Tübingen and the University Hospital Erlangen to assess Internet accessibility and Internet use in patients with psychosomatic disorders.

Specialized clinicians (medical practitioners and psychologists) conducted a diagnostic interview with each patient and assessed the medical history in order to determine treatment options.

Between December 2008 and April 2009, each patient was asked to participate in the survey by filling in a questionnaire before the diagnostic interview. As we were aiming at gaining a first, explorative overview on Internet use by patients with psychosomatic disorders, we did not define any inclusion or exclusion criteria for survey participation.

\section{Questionnaire}

We created a custom-made questionnaire to cover all information relevant to the present 
research question. This questionnaire covers socio-demographic information and details concerning Internet access and use. Six questions were multiple-choice and six were essay-style questions.

Questions concerning Internet access and use first asked if the respective person has Internet access at all. Further questions covered, when applicable, technical equipment, service provider, Internet speed, for what purposes the Internet is used, frequency and duration of weekly Internet use, use for health-related topics, and further requests concerning health-related topics.

Socio-demographic information included gender, age, and place of residence. Then the staff member who conducted the diagnostic interview completed each of their participating patients' main diagnosis.

\section{Statistics}

Unless otherwise indicated, results are either reported as frequencies or as mean \pm standard deviation.

The indicated place of residence was classified according to the German Federal Statistics Agency's following definitions: large city ( $>$ 100,000 inhabitants), medium-sized city ( $>$ 20,000 to 100,000 inhabitants), small town $(>5,000$ to 20,000 inhabitants), and rural area $(<5000$ inhabitants).

Correlations between two continuous variables were analyzed using Pearson correlation (e.g. age and time spent on the Internet). Differences between groups were either examined using Student's $t$-test or one-way ANOVA (in the case of interval data, e.g. age) or by $X^{2}$ test (in the case of nominal or ordinal data, e.g. Internet access). To determine individual group differences when overall differences between groups were significant, post-hoc tests were calculated.

\section{Results}

\section{Patients}

In the assessment period, a total of 334 of the outpatient clinics' first-time patients were
Table I. Basic patient characteristics

\begin{tabular}{lc}
\hline$N$ & 274 \\
Females (\%) & 66 \\
Age (yrs) & $39.5 \pm 10.1$ \\
Place of residence (\%) & 7.8 \\
Rural area & 40.2 \\
Small town & 34.5 \\
Medium-sized city & 7.8 \\
Large city & \\
Diagnostic group (\%) & 23 \\
Mood disorders & 16 \\
Somatoform disorders & 16 \\
Anxiety disorders & 16 \\
Eating disorders & 16 \\
Obesity & 13 \\
Other disorders &
\end{tabular}

asked to participate in the survey. In total 274 patients $(82 \%)$ returned a completed questionnaire and were thus included in the study. Table 1 displays the basic characteristics of the study sample.

Female participants were significantly younger than male participants $(37.8 \pm 12.8$ versus $42.3 \pm 13.2$ years; $p<.01$ ).

Although obesity is currently not recognized as a typical disorder on the psychosomatic spectrum, obese patients constituted a significant diagnostic group in the present survey. This is due to obesity being considered as a condition with a biopsychosocial background often accompanied by psychosomatic comorbidities such as eating, mood, and anxiety disorders. Therefore, university hospitals currently offer multidisciplinary treatment approaches for obesity.

There was a significant age difference between patients from the different diagnostic groups $(F=8.863$, d.f. $=5, p<.01)$, with patients with eating disorders being youngest (28.6 \pm 7.7 years) and patients with somatoform disorders being the oldest (44.2 \pm 14.2 years). The post-hoc test revealed that eating disorder patients significantly differed in age from the other diagnostic groups, while the mean age of the remaining five groups did not significantly differ. 
Table 2. Differences between patients with and without Internet access

\begin{tabular}{lll}
\hline Internet access & Yes & No \\
\hline Number & $204(74.2)$ & $70(25.8)^{* *}$ \\
Females (\%) & 67 & 64 \\
Age (yrs) & $37.2 \pm 12.6$ & $45.5 \pm 12.5^{* *}$ \\
Place of residence (\%) & & \\
Rural area & 8 & 7 \\
Small town & 37 & 49 \\
Medium-sized city & 37 & 30 \\
Large city & 18 & 14 \\
Diagnostic group (\%) & & \\
Mood disorders & 23 & 24 \\
Somatoform & 14 & 23 \\
disorders & & \\
Anxiety disorders & 13 & 23 \\
Eating disorders & 19 & $7 *$ \\
Obesity & 16 & 18 \\
Other disorders & 15 & $6 *$ \\
\hline
\end{tabular}

$* p<.05 ; * * p<.01$.

\section{Questionnaire data}

Internet access. As Table 2 displays, the majority of survey participants reported having Internet access. We found no differences in Internet access availability between areas of residence or females and males. However, Internet access availability differed significantly between diagnostic groups. Patients indicating that they had Internet access were significantly younger than those indicating no access.
Internet use. The survey revealed that patients use the Internet as both a communication device and information pool, as Fig. 1 illustrates.

Sixty percent of the participating patients also reported using the Internet for healthrelated purposes and topics, covering research for disorder-related information, treatment options and clinic contact details as well as selfhelp and discussion forums. This usage pattern was equally present across different diagnostic groups $\left(X^{2}=0.65 ; p>.05\right)$ and both sexes $\left(X^{2}=\right.$ $0.15 ; p>.05)$. Moreover, we found no age difference between patients indicating Internet use for health-related topics and those indicating non-health-related Internet use $(36.9 \pm 12.3$ versus $37.3 \pm 13.0$ years; $p>.05)$.

Patient requests concerning health-related Internet use. The qualitative analysis of the self-written answers concerning Internet-based healthrelated offers highlighted patients' main user requests. Patients wanted more evidence-based, professional information, such as 'recent results of clinical studies on drugs and treatments' or 'expert-certified homepages', that is also understandable to medical laypeople, for instance 'less technical terms' and 'patient-oriented language'. Second, patients requested the possibility to research technical terms, including 'an encyclopedia of medical terms'. Lastly, the

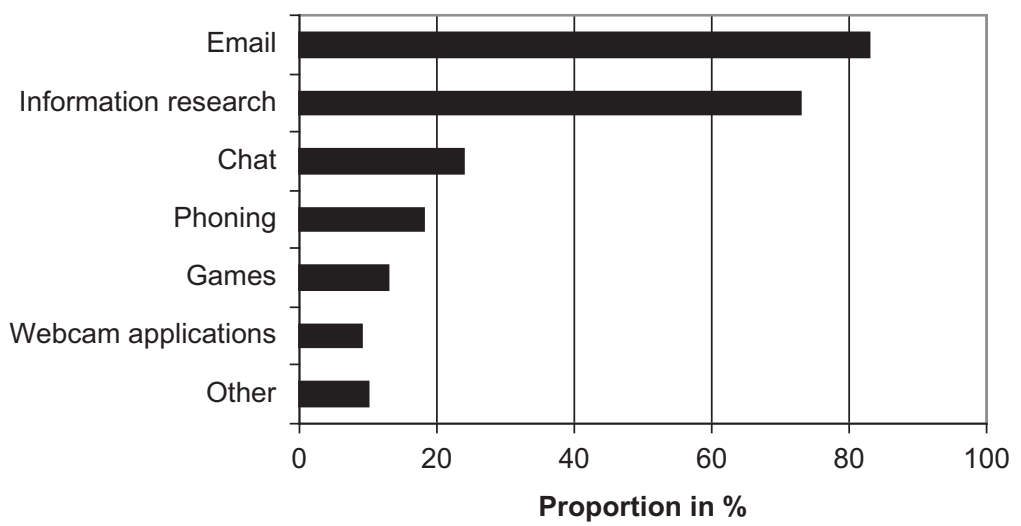

Figure I. Purposes of Internet use $(N=204)$. 
responders expressed interest in receiving Internet-based expert-guided treatments, such as 'therapeutically guided forums'. Furthermore, patients wanted more Internet options to find appropriate treatment institutions and online patient forums and support groups.

\section{Discussion}

Our survey revealed that as many as 74.2 percent of the patients with psychosomatic disorders participating in our survey had Internet access. This accessibility rate is comparable with the German Federal Statistical Office's recent report stating that 73 percent of private German households have Internet service (Statistisches Bundesamt, 2009). The Internet was equally accessible for both sexes and was not reliant on the area of residence. These data suggest that a majority of patients suffering from psychosomatic disorders in Germany have Internet access and that Internet-based health information and health care can reach even those in rural areas. However, the Internet is currently used more by younger individuals, as other surveys previously reported (Buntrock et al., 2007; Gordon et al., 2002; Pereira et al., 2000; Statistisches Bundesamt, 2009). We also found that Internet accessibility differed between diagnostic groups with patients with eating disorders reporting the greatest access. This effect is probably due to the reported age difference between eating disordered patients and other diagnostic groups. Eating disorder onset happens when people are young, therefore patients are typically in their early $20 \mathrm{~s}$.

Most patients with Internet access use broadband technology. Broadband Internet fulfills the technological prerequisites to participate in web-based interventions, including at-home video conferencing. However, with respect to video conferencing technology, only a few survey participants reported currently using webcam applications.

Sixty percent of the patients reported that they use the Internet to search for health-related information. This rate is comparable with other patient groups, for example in a sample of general practice patients (Diaz et al., 2002), cancer patients (Castleton et al., 2010), and patients with psychiatric disorders (Khazaal et al., 2008).

Internet use for health-related topics was independent of gender, age, and diagnostic group. This underlines the Internet's importance for the health-related behavior of patients with psychosomatic conditions, with patients wanting even more evidence-based, professional information. The qualitative analysis additionally revealed the patients' desire for Internet-based expert-guided treatments. Even though an increasing number of studies has been published about online programs offering psychotherapy (Kessler et al., 2009; Mitchell et al., 2008), self-help (Van't Hof et al., 2009) and the aftercare of various conditions and disorders (Golkaramnay et al., 2007), these novel approaches have not yet been disseminated to a broad community of patients. However, patients' expressed desire for such programs demonstrates the need for more structured, expert-guided online interventions and encourages the development of such approaches.

The study was conducted at two university hospitals in Germany, but it is likely that results can be applied to other treatment settings in western countries. A shortcoming of our survey lies in the lack of more detailed demographic information, for example on education and socioeconomic status, which is known to be associated with Internet use behavior (Diaz et al., 2002).

Taken together, our data show the Internet's high potential within future treatment strategies in psychosomatic medicine. Among the patients at our university hospitals, we found a high percentage of broadband Internet, active Internet use for health-related issues, a desire for professional information, and for web-based treatments and consultations. Our findings encourage the development of further innovative Internet-based material for patients with psychosomatic and psychiatric complaints and disorders. 


\section{Competing Interests}

None declared.

\section{References}

Buntrock S, Hopfgarten T, Adolfsson J, Onelöv E and Steineck G (2007) The Internet and prostate cancer patients: Searching for and finding information. Scandinavian Journal of Nephrology 41(5): 367-374.

Castleton K, Fong T, Wang-Gillam A, et al. (2010) A survey of Internet utilization among patients with cancer. Supportive Care in Cancer, 1-8.

Diaz JA, Griffith RA, Ng JJ, Reinert SE, Friedmann PD and Moulton AW (2002) Patients' use of the Internet for medical information. Journal of General Internal Medicine 17(3): 180-185.

Donker T, Van Straten A, Marks I and Cuijpers P (2009) A brief web-based screening questionnaire for common mental disorders: Development and validation. Journal of Medical Internet Research 11(3): e19.

Enck P, Kowalski A, Martens U and Klosterhalfen S (2006) Internet-based assessment of bowel symptoms and quality of life. European Journal of Gastroenterology \& Hepatology 18(12): 12631269.

Evers KE, Prochaska JM, Prochaska JO, Driskell M-M, Cummins CO and Velicer WF (2003) Strengths and weaknesses of health behavior change programs on the Internet. Journal of Health Psychology 8(1): 63-70.

Golkaramnay V, Bauer S, Haug S, Wolf M and Kordy H (2007) The exploration of the effectiveness of group therapy through an Internet chat as aftercare: A controlled naturalistic study. Psychotherapy and Psychosomatics 76(4): 219-225.

Gordon MM, Capell HA and Madhok R (2002) The use of the Internet as a resource for health information among patients attending a rheumatology clinic. Rheumatology 41(12): 1402-1405.

Kessler D, Lewis G, Kaur S, et al. (2009) Therapistdelivered Internet psychotherapy for depression in primary care: A randomised controlled trial. Lancet 374(9690): 628-634.

Khazaal Y, Chatton A, Cochand S, et al. (2008) Internet use by patients with psychiatric disorders in search for general and medical informations. Psychiatric Quarterly 79(4): 301-309.

Kiropoulos LA, Klein B, Austin DW, et al. (2008) Is Internet-based $\mathrm{CBT}$ for panic disorder and agoraphobia as effective as face-to-face CBT? Journal of Anxiety Disorders 22(8): 1273-1284.

Marziali E (2009) E-health program for patients with chronic disease. Telemedicine Journal and E-Health: The Official Journal of the American Telemedicine Association 15(2): 176-181.

Mitchell JE, Crosby RD, Wonderlich SA, et al. (2008) A randomized trial comparing the efficacy of cognitive-behavioral therapy for bulimia nervosa delivered via telemedicine versus face-to-face. Behavior Research and Therapy 46(5): 581-592.

Olsson S, Lymberis A and Whitehouse D (2004) European Commission activities in eHealth. International Journal of Circumpolar Health 63(4): 310-316.

Pereira JL, Koski S, Hanson J, Bruera ED and Mackey JR (2000) Internet usage among women with breast cancer: An exploratory study. Clinical Breast Cancer 1(2): 154-155.

Probst J, Laditka S, Wang J and Johnson A (2007) Effects of residence and race on burden of travel for care: Cross sectional analysis of the 2001 US National Household Travel Survey. BMC Health Service Research 7: 40.

Roberto CA, Grilo CM, Masheb RM and White MA (2010) Binge eating, purging, or both: Eating disorder psychopathology findings from an Internet community survey. International Journal of Eating Disorders 43(8): 724-731.

Simpson S, Barnes E, Griffiths E, et al. (2009) The Bipolar Interactive Psychoeducation (BIPED) study: Trial design and protocol. BMC Psychiatry 12(9): 50.

Statistisches Bundesamt (2009) Private Haushalte in der Informationsgesellschaft 2009. Available at: http://www.destatis.de/jetspeed/portal/cms/Sites/ destatis/Internet/DE/Presse/pm/2009/12/ PD09_464_IKT,templateId=renderPrint.psml.

Teufel M, Giel KE and Zipfel S (2009) Telemedicine and psychotherapy - empathy through distant cyberspace? Psychotherapie, Psychosomatik, Medizinische Psychologie 59(8): 289-290.

Trotter MI and Morgan DW (2008) Patients' use of the Internet for health related matters: A study of Internet usage in 2000 and 2006. Health Informatics Journal 14(3): 175-181.

Van Straten A, Cuijpers P and Smits N (2008) Effectiveness of a web-based self-help intervention for symptoms of depression, anxiety, and stress: Randomized controlled trial. Journal of Medical Internet Research 10(1): e7. 
Van't Hof E, Cuijpers P and Stein DJ (2009) Self-help and Internet-guided interventions in depression and anxiety disorders: A systematic review of metaanalyses. CNS Spectrum 14(2 Suppl. 3): 34-40.
Wisner KL, Logsdon MC and Shanahan BR (2008) Web-based education for postpartum depression: Conceptual development and impact. Archives of Women's Mental Health 11(5-6): 377-385. 